every case, the lesion echoes showed high fluctuating amplitude and widtl during coagulation procedure. Lesion echoes were observed up to one week after the surgery. They maintained stable duplicate echoes during the period.

It is our suggestion that the ultrasonic examination of the stereotaxic lesion is useful to note accidental hemorrhage in the lesion of a reconvalescent patient. It is also a great aid for this purpose to check the midline echo at the same time.

\title{
4. Basic Researches for Stereotaxic Surgery
}

II Changes in and around the Turget Point

Shinken Kuramoto, Mitsuo Watanabe, Ryoichi Oonaka

and Toshiro TACHIBANA

Dept. of Surgery, Kurume University Medical School

\section{Considerations on Hemiplegia due to} Stereotaxic Thalamotomy

\author{
Isao MIURA \\ Dept. of Psychiatry, Keio University
}

Nobuo Yoshil, Shigetomi Miyagawa and Toshiro Shizawa

Dept. of Neurosurgery, Keio University

Sakae FufuDA

Dept. of Neurosurgery, Shizuoka Red Cross Hospital 